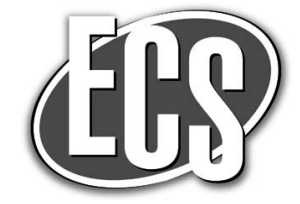

Jes Focus Issue Honoring Allen J. Bard

\title{
Probing Passivating Porous Films by Scanning Electrochemical Microscopy
}

\author{
Christian Kuss, ${ }^{*}$ Nicholas A. Payne, ${ }^{*}$ and Janine Mauzeroll ${ }^{\mathrm{Z}}$ \\ Laboratory for Electrochemical Reactive Imaging and Detection of Biological Systems, McGill University, Montreal, \\ QC H3A OB8, Canada
}

\begin{abstract}
Porous films are ubiquitous in electrochemistry. They frequently form on active electrodes due to the precipitation of insoluble reaction products. They can have beneficial effects, like the protection from electrochemical corrosion, or be of parasitic nature, as in the poisoning of fuel cell air cathodes. The effects of such layers on the electrochemical response of the substrate can be probed by Scanning Electrochemical Microscopy (SECM). Herein, we present modifications to the conventional analytical expressions for SECM microelectrode approach curves, to account for the effects of a porous layer. The modified expressions can be used to fit experimental approach curves and obtain film thickness and porosity parameters. Their performance is demonstrated through comparison to results obtained by finite element modeling, and by fitting experimental approach curves over well-defined filter membranes.

(C) The Author(s) 2015. Published by ECS. This is an open access article distributed under the terms of the Creative Commons Attribution 4.0 License (CC BY, http://creativecommons.org/licenses/by/4.0/), which permits unrestricted reuse of the work in any medium, provided the original work is properly cited. [DOI: $10.1149 / 2.0131604$ jes] All rights reserved.
\end{abstract}

Manuscript submitted September 30, 2015; revised manuscript received November 23, 2015. Published December 5, 2015. This paper is part of the JES Focus Issue Honoring Allen J. Bard.

Surface passivation reduces the accessibility and electrochemical activity of electrodes through the deposition of an insulating layer. It plays a crucial role in almost every aspect of electrochemical research, including electrochemical corrosion, ${ }^{1}$ fuel cells, ${ }^{2}$ photoelectrochemical water splitting, ${ }^{3}$ and electrochemical energy storage. ${ }^{4}$ Surface passivation can be a desired effect, for example in protecting electrodes from corrosion, or have a parasitic effect, that reduces the performance of electrochemical systems. As such, much time and effort is spent on the characterization of passivating films, e.g. corrosion products, ${ }^{5,6}$ the solid electrolyte interface ${ }^{7}$ or passivating layers on fuel cell catalysts. ${ }^{2}$

In many cases, the degree to which a film is passivating is determined by its porosity and thickness. Both values are difficult to determine experimentally, and most frequently ex situ electron microscopy in combination with focused ion beam cutting and milling is employed. ${ }^{5}$ Studying properties of porous films in situ, for example measuring the change of film thickness during the corrosion process, is more complicated. Electrochemical impedance spectroscopy can be used to extract parameters, such as resistance through a surface film, for an assessment of the passivating capability, but requires the complex analysis of impedance spectra. ${ }^{6,8}$

Professor Allen J. Bard, to whom the present issue is dedicated, introduced an original technique to study microscopic properties of electrochemical systems in 1989, Scanning Electrochemical Microscopy (SECM). ${ }^{9}$ Among many other applications, this technique has been applied to the study of porous films. Over conducting substrates, substrate generation / tip collection mode has recently been used to study porous Pt substrates. ${ }^{10}$ In feedback mode, de Smet and co-workers studied the response of conducting silicon substrates in detail, suggesting models to describe the feedback behavior. ${ }^{11}$ SECM studies of insulating, inactive porous films, on the other hand, have been mainly qualitative in nature. In corrosion research, SECM has been used to investigate the corrosion product film, concluding about the porosity and barrier efficiency of these layers. ${ }^{12,13}$ In lithium-oxygen battery research, SECM was used to investigate oxygen transport through the gas diffusion electrode. ${ }^{4}$ Quantitative analysis of pores by SECM has only been achieved over membranes with pore dimensions larger than the microelectrode, at which single pores could be imaged and studied separately. ${ }^{14,15}$ Quantification of the collective effect of many pores in an inactive porous film, and extraction of porous film parameters, such as porosity and thickness by SECM is not yet reported. In the

\footnotetext{
*Electrochemical Society Student Member.
}

${ }^{\mathrm{z}}$ E-mail: janine.mauzeroll@mcgill.ca present study, we describe a method for the quantification of thickness and porosity of inactive porous films using SECM approach curves.

\section{Experimental}

Materials.- A silver cylinder (Goodfellow Cambridge Limited, Huntingdon, England) of diameter $1 \mathrm{~cm}$ was fixed in cold mounting epoxy (Epofix, Streurs, Ontario, Canada) and the cross sectional area was exposed with a coarse polishing step with $\mathrm{SiC}$ paper $(800,1200$, 4000 grit, Streurs). The substrates were polished to a mirror finish with $1,0.3,0.1$ and $0.05 \mu \mathrm{m}$ alumina powder on polishing cloths (Streurs) followed by sonication for 30 minutes in nanopure water (Milli-Q water $18.2 \mathrm{M} \Omega-\mathrm{cm}$, Millipore, USA). Two types of membrane filters were used in the SECM experiments. Designated as membrane A was a polycarbonate membrane filter (Nucleopore, Canada) with manufacturer-specified thickness of $11 \mu \mathrm{m}$, porosity of 0.16 and pore size of $1 \mu \mathrm{m}$. A mixed-cellulose esters membrane filter (Millipore) with thickness of $150 \mu \mathrm{m}$, porosity of 0.75 and pore size $0.22 \mu \mathrm{m}$ was designated as membrane $\mathrm{B}$.

Instrumentation.- SECM approach curve measurements were performed with an ElProscan 1 system (HEKA, Germany; bipotentiostat model PG340). A Pt microelectrode (ME) (diameter $=25 \mu \mathrm{m}$, $\mathrm{R}_{\mathrm{g}}=3$ ) prepared using the literature procedure, ${ }^{16} \mathrm{a} \mathrm{Ag} / \mathrm{AgCl}$ quasireference electrode prepared following literature ${ }^{17}$ using a $1.0 \mathrm{~mm}$ diameter, annealed $99.99 \% \mathrm{Ag}$ wire, (Goodfellow) and a $0.5 \mathrm{~mm}$ diameter Pt wire counter electrode (99.99\%, Goodfellow) were employed in an aqueous solution of $1 \mathrm{mM} \mathrm{Ru}\left(\mathrm{NH}_{3}\right)_{6} \mathrm{Cl}_{3}$ (Sigma Aldrich, Oakville, Canada) in $0.1 \mathrm{M} \mathrm{NaCl}$ (Sigma Aldrich) for SECM measurements. The substrates for the SECM approach curves were prepared by placing a pre-wetted filter membrane over the silver substrate and securing with a ring cut from cured epoxy (Struers). Consistency of the steady state current over time was confirmed through cyclic voltammetry before and after each approach curve. All feedback approach curves were performed at $1 \mu \mathrm{m} / \mathrm{s}$ with the ME polarized at $-400 \mathrm{mV}$ vs. $\mathrm{Ag} / \mathrm{AgCl}$ in order to reduce the redox mediator $\left(\mathrm{Ru}\left(\mathrm{NH}_{3}\right)_{6} \mathrm{Cl}_{3}\right)$ at a diffusion-limited rate.

Filter membranes were imaged using a Hitachi SU3500 Variable Pressure scanning electron microscope (SEM) with images taken at a pressure of $30 \mathrm{~Pa}$ due to low conductivity of the membranes. The thicknesses of the membrane filters were measured using a micrometer thickness gauge (Mitutoyo, Canada). Provided errors are statistical errors, assuming a Student's t distribution, for a confidence level of $95 \%$, based on 6 separate measurements. 
Fitting approach curves to analytical approximations.- Approach curves were fitted using the trust-region-reflective least squares fitting algorithm in Matlab R2015a. The Matlab function, describing the modified expression for approach curves above isotropically porous membranes can be found as part of the supplementary material. The electrode $\mathrm{R}_{\mathrm{g}}$ was determined through fitting of an approach curve above plastic substrate to Eq. 1 and was confirmed by optical microscopy. Subsequent approach curves above membrane covered silver substrates were fitted, using the normalized substrate rate constant as fitting parameters for Eq. 5 (membrane A), and film porosity and film thickness as fitting parameters for Eqs. 6 and 7 (membrane B), assuming a fast kinetics at the underlying substrate $\left(\kappa^{s u b}=10^{10}\right)$. The fitting was also allowed to adjust for small variations in the steady state current by $\pm 2 \%$. All provided errors are statistical errors, assuming a Student's t distribution, for a confidence level of $95 \%$, based on 6 independent approach curves.

Finite element modeling. - Finite element models were established in the COMSOL 5.0 Multiphysics software package making use of the Chemical Reaction Engineering Module. The model was set up to solve Fick's equations, neglecting any migration effects, in a 2-dimensional axially symmetric system. Within the porous film, the bulk diffusion coefficient was reduced by a porosity-tortuosity factor. A more detailed description of the model can be found in the supplementary material.

\section{Results and Discussion}

Derivation of analytical approximations. - A very common and useful characterization method in SECM is the feedback mode, in which a redox mediator is added to the electrolyte solution. ${ }^{9} \mathrm{By}$ oxidizing or reducing the mediator at the microelectrode at a diffusion limited rate, it is possible to probe local changes in mediator flux, which can be due to changes in diffusion geometry and local mediator concentration. When studying a substrate with feedback mode SECM, two main effects are commonly considered: i. Negative feedback is caused by reduced mass transport to the microelectrode due to the hindrance of diffusion by the presence of a large substrate; ii. Positive feedback originates from the regeneration of the redox mediator by an electrochemically active substrate, causing a local increase in the concentration of available mediator. These effects lead respectively to reduced and increased mediator flux to the microelectrode, which determines the measured current. Such behavior is commonly assessed by moving the microelectrode from the bulk solution closer to the surface while measuring the current, recording an approach curve. In pure negative feedback, the current reduces to zero, whereas in pure positive feedback the current rises to infinity, when the electrode is short-circuited to the substrate provided the electrode surface is parallel to the substrate. This can be largely reproduced by analytical approximations, which were reviewed in detail by Lefrou and Cornut in $2010 .{ }^{18}$ For comparability of currents between different microelectrodes, these mathematical expressions use tip currents $\left(N i_{T}\right)$ normalized to the steady state current in the bulk electrolyte with distances $(L)$ and glass sheath radii $\left(R_{g}\right)$ normalized to radius of the electroactive area of the microelectrode. Normalized substrate rate constants $(\kappa)$ are calculated by multiplying the substrate rate constant by the radius of the electroactive area and dividing by the diffusion coefficient of the redox mediator. In there, the negative feedback current response takes on the form:

$$
\begin{aligned}
& N i_{T}^{i n s}\left(L, R_{g}\right) \\
& =\frac{2.08 \cdot R_{g}^{-0.358} \cdot\left(L-\frac{0.145}{R_{g}}\right)+1.585}{2.08 \cdot R_{g}^{-0.358} \cdot\left(L+2.3 \cdot 10^{-3} \cdot R_{g}\right)+1.57+\frac{\ln R_{g}}{L}+\frac{2}{\pi R_{g}} \cdot \ln \left(1+\frac{\pi R_{g}}{2 L}\right)}
\end{aligned}
$$

Whereas the positive feedback current response can be modelled as:

$$
\begin{aligned}
N i_{T}^{\text {cond }}\left(L, R_{g}\right)= & \alpha\left(R_{g}\right)+\frac{1}{\beta\left(R_{g}\right)} \cdot \frac{\pi}{4 \operatorname{ArcTan} L} \\
& +\left(1-\alpha\left(R_{g}\right)-\frac{1}{2 \beta\left(R_{g}\right)}\right) \cdot \frac{2}{\pi} \operatorname{ArcTan} L
\end{aligned}
$$

With:

$$
\begin{array}{r}
\alpha\left(R_{g}\right)=\ln 2+\ln 2 \cdot\left(1-\frac{2}{\pi} \operatorname{ArcCos} \frac{1}{R_{g}}\right) \\
-\ln 2 \cdot\left[1-\left(\frac{2}{\pi} \operatorname{ArcCos} \frac{1}{R_{g}}\right)^{2}\right] \\
\beta\left(R_{g}\right)=1+\frac{0.1380}{\left(R_{g}-0.6723\right)^{0.8686}}
\end{array}
$$

On some substrates, intermediate current responses can be recorded, showing contributions from both feedback effects, when regeneration at the substrate is slow i.e. intermediary kinetics between positive and negative feedback which therefore limits the feedback current during an approach curve. If this regeneration behaves according to first order reaction kinetics, an analytical approximation can also be found for this case: ${ }^{19}$

$$
\begin{aligned}
& N i_{T}\left(L, R_{g}, \kappa\right)=N i_{T}^{\text {cond }}\left(L+\frac{1}{\kappa}, R_{g}\right) \\
& +\frac{N i_{T}^{i n s}\left(L, R_{g}\right)-1}{\left(1+2.47 \cdot R_{g}^{0.31} \cdot L \cdot \kappa\right)\left(1+L^{0.06 R_{g}+0.113} \kappa^{-0.0236 R_{g}+0.91}\right)}
\end{aligned}
$$

These equations have been a valuable addition to the toolset of SECM, allowing quantitative analysis of many experimental results. However, they are insufficient when the approach curve behavior is affected by a porous layer on the substrate.

Effect of a porous layer on negative feedback. - If an electrochemically inactive micro-porous layer, with porosity $P$ and normalized thickness $B$ (normalized to the radius of the electroactive area), is present on an electrochemically inactive substrate, the microelectrode current does not reduce to zero when it touches the porous surface, but the current drops to a finite value instead. This is because the mediator can diffuse through the porous layer to the microelectrode tip, giving rise to a measurable current. As the distance between microelectrode and substrate becomes larger, the contribution of diffusion through the porous layer to the microelectrode becomes smaller. Nevertheless, when such an approach curve is analyzed with the introduced analytical approximation (Eq. 6), it is consequently assumed to show a mediator regenerating surface, even if the surface is completely inactive.

In order to compensate for the diffusion of the redox mediator through the porous film for quantitative analysis, we used a simple modification of the established analytical approximation (Eq. 1) for negative feedback. Diffusional flux is linearly dependent on the diffusion coefficient, which, in turn, depends linearly on the porosity in the porous media. In a first approximation, we can thus approach the current response behavior above the porous layer as a linear composition of the two extreme cases of porosity, zero and one. In the case of a porous layer with porosity approaching zero, the approach curve resembles Eq. 1, with $N i_{T}^{i n s}\left(L, R_{g}\right)$, since the porous layer can be approximated to be an inactive filled solid. In the case of a porous layer with porosity approaching one, the approach curve resembles Eq. 1, with $N i_{T}^{i n s}\left(L+B, R_{g}\right)$, since the porous layer can be approximated to be bulk electrolyte. The negative feedback equation with a porous layer present can thus be modified to:

$$
\begin{aligned}
N i_{T}^{\text {ins,por }}\left(L, R_{g}, P, B\right)= & P \cdot N i_{T}^{i n s}\left(L+B, R_{g}\right) \\
& +(1-P) \cdot N i_{T}^{i n s .}\left(L, R_{g}\right)
\end{aligned}
$$


Effect of a porous layer on positive feedback.- The cycling of mediator oxidation and reduction between microelectrode and substrate can be affected by a porous layer as well. In this case, the diffusional flux to and from the substrate is reduced by the lower apparent diffusion coefficient of the mediator in the porous layer. Assuming a porous layer over a substrate that regenerates the mediator at diffusion-limited kinetics, the measured currents by the microelectrode would appear smaller than above the substrate without overlying porous layer. As such, the conventional analytical approximations would give too small a kinetic constant for the substrate.

A simple, one-dimensional, diffusional model can provide a first approximation for this effect of a porous layer on the positive feedback response. It has already been successfully applied in analyzing approach curves over corrosion product covered magnesium surfaces. ${ }^{20}$ This diffusion of species from the substrate through the film can be accounted for through the introduction of an apparent normalized rate constant, $\kappa^{\text {por }}$, obtained from a modification of the normalized substrate rate constant $\kappa^{\text {sub }}$ :

$$
\kappa^{p o r}\left(P, B, \kappa^{s u b}\right)=\frac{P}{B+\frac{P}{\kappa^{s u b}}}
$$

Anisotropy and tortuosity. - It is important to note that for both positive and negative feedback modifications, the isotropy and tortu- osity of the porous layer play a role. The equations (Eqs. 6 and 7) were established, considering isotropic conditions, and the assumptions that have been made are not valid in anisotropic films. However, there are two special cases of anisotropically-porous films for which the established equations suffice in obtaining good fits. In the case of pores that are perfectly parallel to the substrate, there is no electrolytic connection between substrate and bulk solution. Hence, such surface would react as ideal insulator, and Eq. 1 is applicable. When the pores are perfectly perpendicular to the substrate, mediator can only diffuse to and from the surface, but not parallel to the surface. Consequently, such a porous film would mainly affect the positive feedback contribution to the approach curve, but have no effect on the negative feedback.

The isotropic tortuosity of the film has not been considered, as it is in many cases much less influential than the porosity on the diffusion. In cases were tortuosity is an important factor to consider, this factor is included in the porosity such that $P=P^{\prime} / \tau$, where $P$ is the porositytortuosity factor, $P^{\prime}$ is the physical porosity of the film and $\tau$ is the tortuosity.

Comparison to finite element modelling. - The introduced expressions serve as a first approximation to investigate the effect of a porous layer on SECM feedback curves. More accurate solutions for such complex systems can commonly only be achieved with suitably opti-

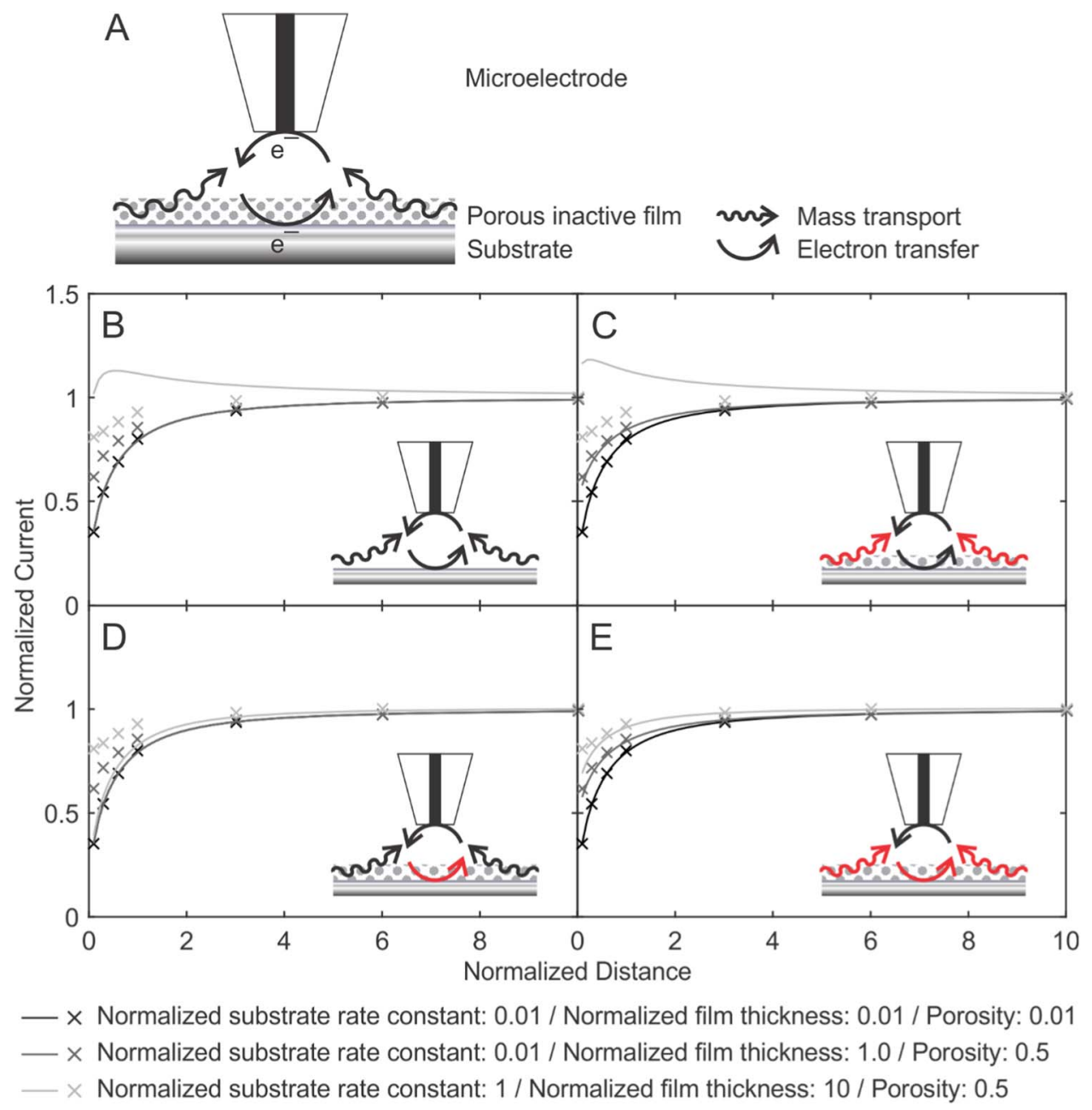

Figure 1. Negative and positive feedback contributions of an inactive porous film. Schematic of the contributions (A). Examples of the effect of the porous layer on the current response for three different cases, and the ability to reproduce this behavior for the literature analytical approximation (Eq. 5(18)) (B), and its modifications (highlighted in red in the inlayed schemes), accounting for negative feedback (Eq. 6) (C), positive feedback (Eq. 7) (D), and both contributions (E) of the porous layer. In each schematic, the crosses represent the output from finite element modeling, while the lines represent the fits to the specified analytical approximation. 
mized finite element modeling, at which Fick's diffusion equations are solved iteratively. In order to quantify the remaining error between the proposed analytical estimation of the effect of porous layers and the iteratively solved results, we compared approach curves obtained with the Multiphysics software package COMSOL to those obtained using above described equations for different porosities, film thicknesses, and substrate rate constants. Figure 1 shows three different cases of model systems, applying no modification to the literature analytical expression Eq. 5 and modifications for the negative (Eq. 6), positive (Eq. 7) and both feedback contributions of the porous layer to Eq. 5. At very thin films, the porous layer effect is not strong, as can be seen in the first examined case (Figure 1, black curve). Consequently, the literature analytical expression can well reproduce the modelled current response. The increased current over a substrate of very low reactivity with a porous layer present can be compensated well with the negative feedback modification (Figure 1, dark gray curve). However, substrates with high rate constant (Figure 1, light gray curve) show a reduction in current when a porous layer is present, which can only be captured when both, negative and positive, feedback modifications are considered. Given the minimal modifications introduced, a surprisingly good fit can be obtained for all three considered cases, if both negative and positive feedback effects are taken into account.

This comparison can be expanded to a more complete set of cases, as displayed in Figure 2. Represented is the errors between finite element modeling and the analytical approximations both corrected and uncorrected as indicated for a wide variety of cases. For each
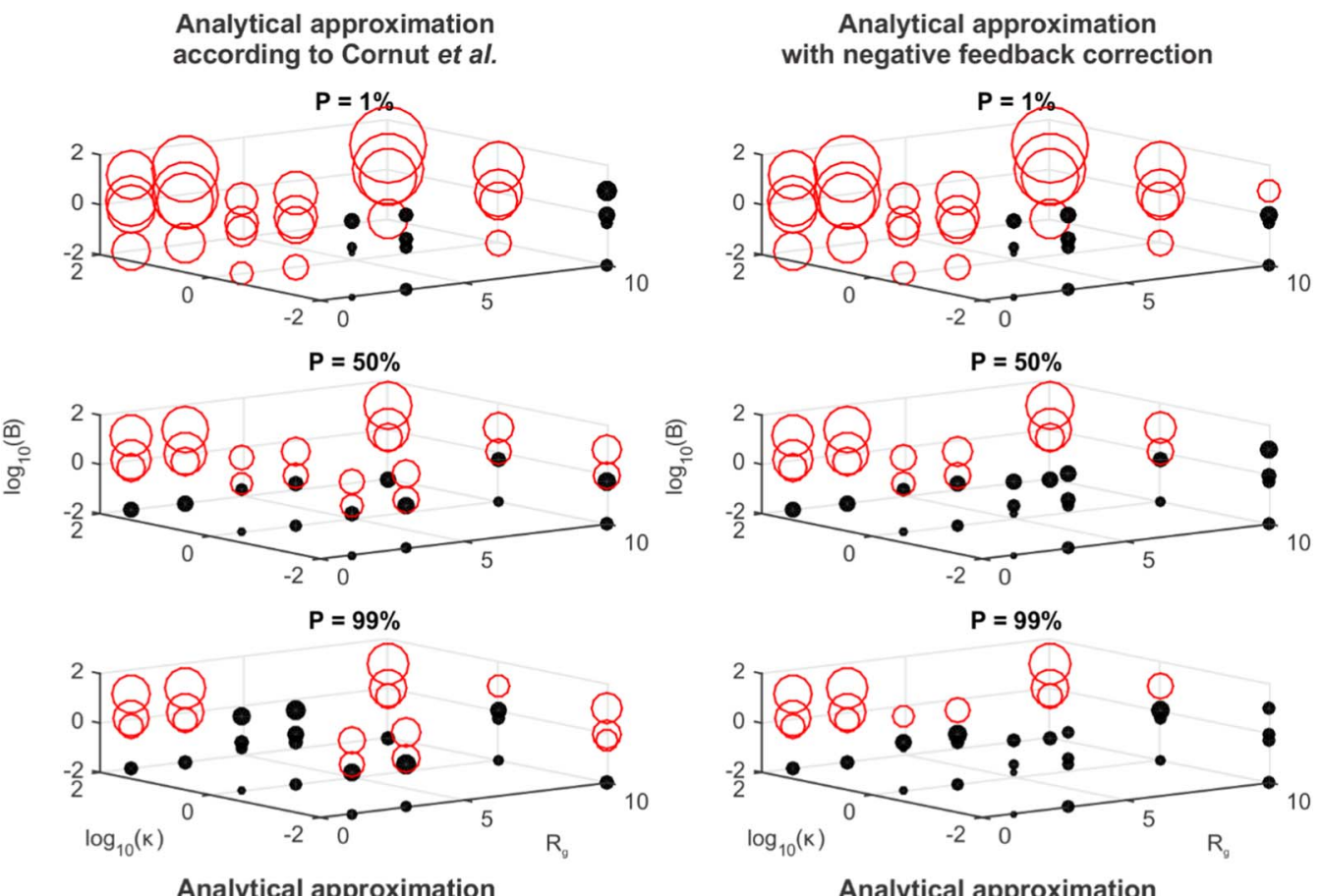

Analytical approximation

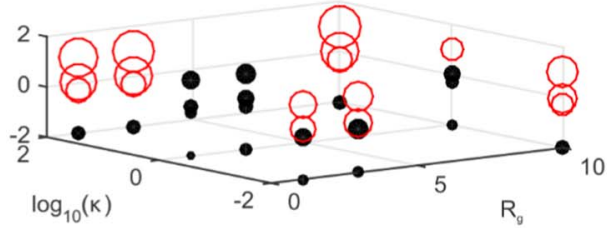

Analytical approximation with positive feedback correction

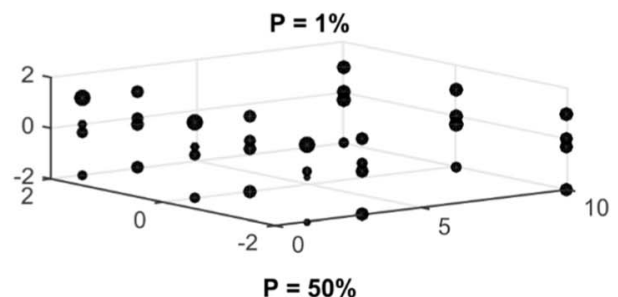

with negative and positive feedback correction
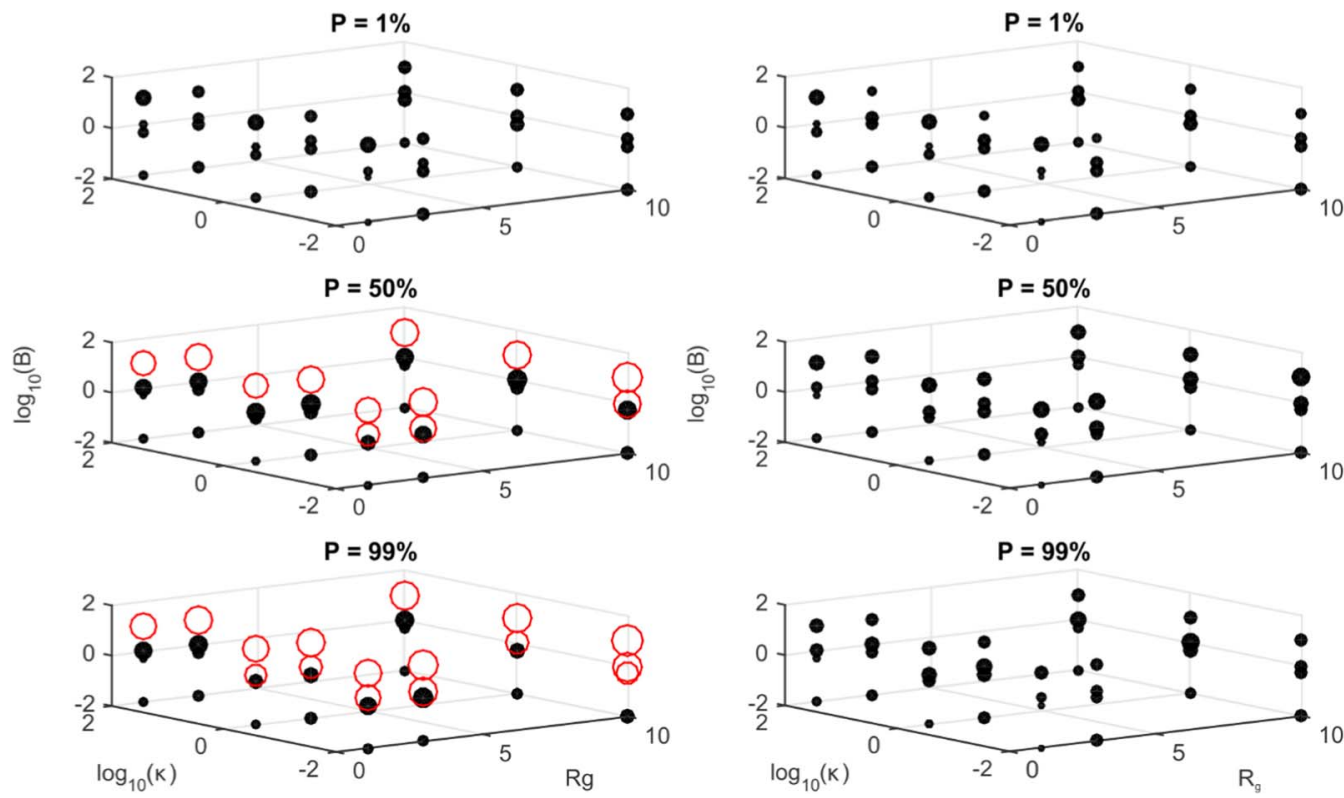

Figure 2. Mean relative deviations between approach curves derived from analytical expressions and finite element models for films of different porosities $\mathrm{p}$, substrate rate constants $\kappa$, electrode parameters $\mathrm{R}_{\mathrm{g}}$ and normalized film thicknesses $\mathrm{B}$. The size of the black spots correlates to the mean current deviation per approach curve. Red circles indicate deviations larger than $10 \%$. 

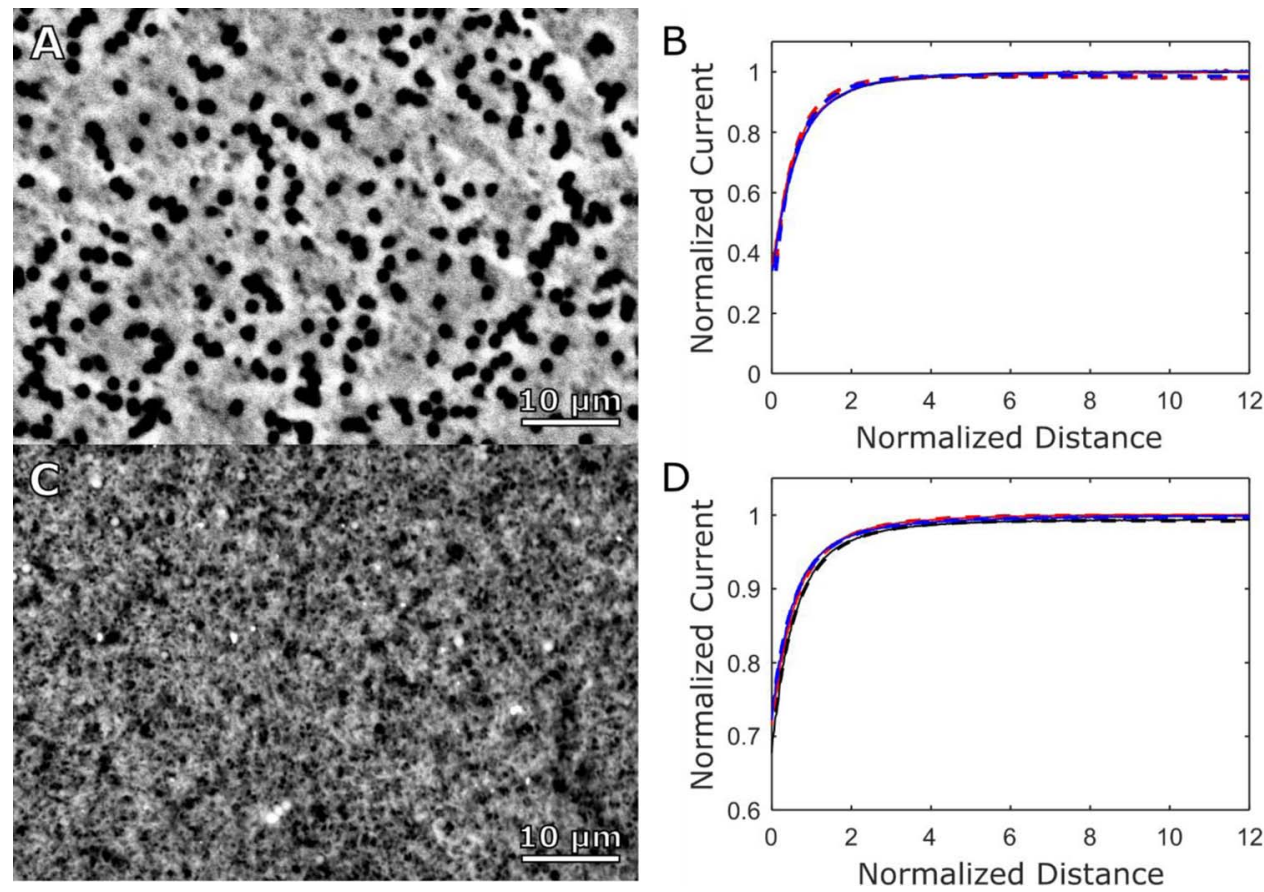

Figure 3. SEM images (A, C) and approach curves (B, D) of filter membranes A (A, B) and B (C, D). Experimental data are represented by solid and fits by dashed lines.

type of fit, three graphs are presented at three values of porosity $(\mathrm{P})$ as specified. Each graph consists of data points at three values of electrode $\mathrm{R}_{\mathrm{g}}$ and substrate rate constant $(\kappa)$ and four values of film thickness (B) giving a total of 108 examples. At each data point, the size of the point, or circle, is a measure of the error between the FEM and indicated analytical approximation. For clarity, fits which gives an error greater than $10 \%$ are colored red.

As can be seen, the negative feedback contribution plays a greater role and reduces errors more significantly when increasing film porosities, and decreasing rate constants whereas positive feedback contributions are more important at low film porosities, and high substrate rate constants. Increasing the film porosity allows for enhanced lateral diffusion of species through the film and enhances the need for the negative feedback correction (Eq. 6), while reducing the porosity limits the contribution of the negative feedback correction. Instead, the effect significantly modifies the apparent rate constant (Eq. 7) thus increasing the need for the positive feedback correction. In the same way that the substrate rate constant can be used to calculate the currents from intermediate surface kinetics based on negative and positive feedback currents using Eq. 5, it also directly shows the significance of the positive and negative feedback corrections. At high rate constants, the surface provides positive feedback which dominates the currents measured while at low rates, negative feedback is much more significant.

When both contributions are included in the analytical approximation, mean deviations between the introduced approximations and the finite element model remain consistently below $7 \%$ per approach curve, with a mean over all approach curves of only $1.8 \%$. Since finite element modelling is not absolutely accurate and in the present case exhibits itself errors of at least $2 \%$, such low errors of the proposed analytical expressions are very promising.

Fitting experimental probe approach curves.- In order to test the applicability of the derived expressions in an experimental setting, microelectrode approach curves were recorded over silver, covered by two types of filter membranes of defined thickness and porosity. Membrane A is of electrode radius thickness, and exhibits anisotropic pores perpendicular to the surface, while membrane $\mathrm{B}$ is a thick and isotropically porous filter membrane (Figures 3A and 3C). As such, the positive feedback effect can be isolated with membrane $A$, while membrane $\mathrm{B}$ affects the approach curve in positive and negative feedback contributions.

Since membrane A only affects the measured normalized apparent rate constant $\kappa^{\text {por }}$, it can be fitted directly to the literature analytical expression Eq. 5 (Figure 3B). Given either porosity or thickness, the other parameter can be calculated from the extracted normalized apparent rate constant with Eq. 7. The obtained fits return a normalized rate constant of $0.19 \pm 0.02$. Taking into account the manufacturer's porosity of 0.16 , this results in a film thickness of $(10.5 \pm 1.1) \mu \mathrm{m}$, which agrees within the error with the manufacturer specified value of $11 \mu \mathrm{m}$ (Table I).

Membrane B approach curves were fitted, using the two introduced modifications Eqs. 6 and 7. Extraordinarily good overlay between fitted and experimental approach curves was achieved for all recorded approach curves (Figure 3D). These fits yield a film thickness of (141 $\pm 21) \mu \mathrm{m}$ and a porosity of $0.83 \pm 0.02$, in good agreement with manufacturer specifications and non-electrochemical measurements (Table I).

Table I. Comparison between film parameters from manufacturer specifications, thickness gauge measurement and SECM approach curve fits.

\begin{tabular}{cllll} 
& & Manufacturer & Thickness gauge & SECM approach curves \\
\hline Membrane A & thickness & $11 \mu \mathrm{m}$ & $(13.5 \pm 0.1) \mu \mathrm{m}$ & $(10.5 \pm 1.1) \mu \mathrm{m}$ \\
& porosity & 0.16 & - & $0.16(\mathrm{not}$ fitted \\
thickness & $150 \mu \mathrm{m}$ & $(155 \pm 1) \mu \mathrm{m}$ & $(141 \pm 21) \mu \mathrm{m}$ & $0.83 \pm 0.02$
\end{tabular}




\section{Conclusions}

The presented, simple modifications to the analytical expressions for SECM approach curves have demonstrated the ability to quantitatively probe porous insulating films over conducting substrates. The significant improvement of the fits to finite element models of porous layers over solid electrodes have shown that the expressions are applicable for a wide variety of parameters. The new expressions were successfully extended to an experimental investigation of two porous films of varying morphology. Modification of the positive feedback contribution to the approach curves by an anisotropicallyporous membrane was performed, and the obtained fitted film thickness shows excellent agreement to the manufacturer's value. A thick, isotropically-porous membrane, that affects both positive and negative feedback, has also shown to be very well fitted by the corrections to the analytical approximations and provide good agreement with data provided by the manufacturer. The introduced modifications provide a new fitting routine for the SECM toolbox, thus further enhancing the reach and impact of SECM in electrochemistry and materials science. With them, ubiquitous porous layers can be probed in situ, allowing quantification of the development of thickness and porosity of growing films on electrodes.

\section{Acknowledgments}

The authors acknowledge funding by General Motors Canada and the Natural Sciences and Engineering Research Council of Canada (NSERC, 140577928).

\section{References}

1. L. Tomcsányi, K. Varga, I. Bartik, H. Horányi, and E. Maleczki, Electrochimica Acta, 34, 855 (1989)
2. S. Koch, M. Mogensen, P. V. Hendriksen, N. Dekker, and B. Rietveld, Fuel Cells, 6 117 (2006)

3. R. Liu, Z. Zheng, J. Spurgeon, and X. Yang, Energy \& Environmental Science, 7, 2504 (2014).

4. P. Schwager, D. Fenske, and G. Wittstock, Journal of Electroanalytical Chemistry, 740, 82 (2015).

5. M. Danaie, R. M. Asmussen, P. Jakupi, D. W. Shoesmith, and G. A. Botton, Corrosion Science, 77, 151 (2013).

6. M. J. Hwang, E. J. Park, W. J. Moon, H. J. Song, and Y. J. Park, Corrosion Science, 96, 152 (2015).

7. S. Zhang, M. S. Ding, K. Xu, J. Allen, and T. R. Jow, Electrochemical and Solid-State Letters, 4, A206 (2001).

8. G. Bolat, D. Mareci, R. Chelariu, J. Izquierdo, S. González, and R. M. Souto, Electrochimica Acta, 113, 470 (2013).

9. A. J. Bard, F. R. F. Fan, J. Kwak, and O. Lev, Analytical Chemistry, 61, 132 (1989).

10. Y.-B. Cho, C. Lee, and Y. Lee, Journal of The Electrochemical Society, 162, H792 (2015).

11. L. C. P. M. de Smet, H. Zuilhof, E. J. R. Sudhölter, G. Wittstock, M. S. Duerdin L. H. Lie, A. Houlton, and B. R. Horrocks, Electrochimica Acta, 47, 2653 (2002).

12. Y. Xia, F. H. Cao, L. R. Chang, W. J. Liu, and J. Q. Zhang, Gaodeng Xuexiao Huaxue Xuebao/Chemical Journal of Chinese Universities, 34, 1246 (2013).

13. Y. Xia, F. Cao, W. Liu, L. Chang, and J. Zhang, International Journal of Electrochemical Science, 8, 3057 (2013).

14. R. Thakar, R. Zakeri, C. A. Morris, and L. A. Baker, Analytical Methods, 4, 4353 (2012).

15. M. Shen, R. Ishimatsu, J. Kim, and S. Amemiya, Journal of the American Chemical Society, 134, 9856 (2012).

16. L. Danis, D. Polcari, A. Kwan, S. M. Gateman, and J. Mauzeroll, Analytical Chemistry, 87, 2565 (2015)

17. T. J. Smith and K. J. Stevenson, in Handbook of Electrochemistry, C. G. Zoski, Editor, p. 73, Elsevier, Amsterdam (2007).

18. C. Lefrou and R. Cornut, ChemPhysChem, 11, 547 (2010).

19. R. Cornut and C. Lefrou, Journal of Electroanalytical Chemistry, 621, 178 (2008).

20. P. Dauphin-Ducharme, C. Kuss, D. Rossouw, N. A. Payne, L. Danis, G. A. Botton, and J. Mauzeroll, Journal of the Electrochemical Society, 162, C677 (2015). 